\title{
IL-17E activates M2 macrophages to produce IL-8 in a p38 dependent manner and favors the recruitment of neutrophils in psoriatic skin
}

\author{
Luisa Senra ${ }^{1}$, Romaine Stalder ${ }^{1}$, Wolf-Henning Boehncke ${ }^{1,2}$, Nicolò C. Brembilla ${ }^{1}$. \\ ${ }^{1}$ Department of Pathology and Immunology, University of Geneva, Geneva, Switzerland \\ ${ }^{2}$ Division of Dermatology and Venereology, University Hospitals of Geneva, Geneva, Switzerland
}

\section{Introduction}

\section{Results}

\section{Ex-vivo}

A. Expression of IL-17E in normal, lesional and non lesional psoriatic skin. B. Expression of IL-17E (red), CD68, IL-17RB (IL-17E specific receptor subunit) and clathrin (green) in lesional skin. C. Correlation between the number of IL-17E+ cells and the number of MPO+ neutrophils or CD3+ $\mathrm{T}$ cells in lesional skin.

\section{In-vitro}

A. IL-17RB expression assessed by qPCR in M0, M1 and M2 monocyte derived macrophages. B. M2 macrophages were stimulated with IL17E. Cytokines/chemokines assessed by qPCR. C. Detection of signaling proteins in $\mathrm{M} 2$ macrophages stimulated with IL-17E for the indicated time points. D. IL- 8 production by ELISA upon treatment with IL-17E alone or in presence of SB202190 or JSH 23. E. Neutrophil chemotaxis assessed in Transwell chamber using supernatants of M2 macrophages alone, in presence of anti-IL-8 antibody or SB 202190.

\section{In-vivo}

A. H\&E and IF staining (Ly6G (green) and CD3 (red)) of back skin of BALB/c mice after two intradermal injections of IL-17E or saline in two consecutive days. B. Quantification of immune cells infiltrating in the skin by FACS. C. Anti-IL17E antibody and respective isotype control were injected i.p. 24h before applying tape-strapping on the back skin of mice. Quantification of immune cells infiltrating in the skin by FACS.
Psoriasis vulgaris is a common chronic recurrent immune-mediated skin disease. As of now, neutralization of Th17/IL23 pathway via blockade of IL-17A or its receptor subunit IL-17RA, represents the most effective approach to treat the disease. In this study we investigated the biological effects of IL-17E, an isoform of the IL-17 family that also signals via the receptor subunit IL-17RA.

IL-17E is overexpressed in psoriasis and positively correlates with infiltrating neutrophils
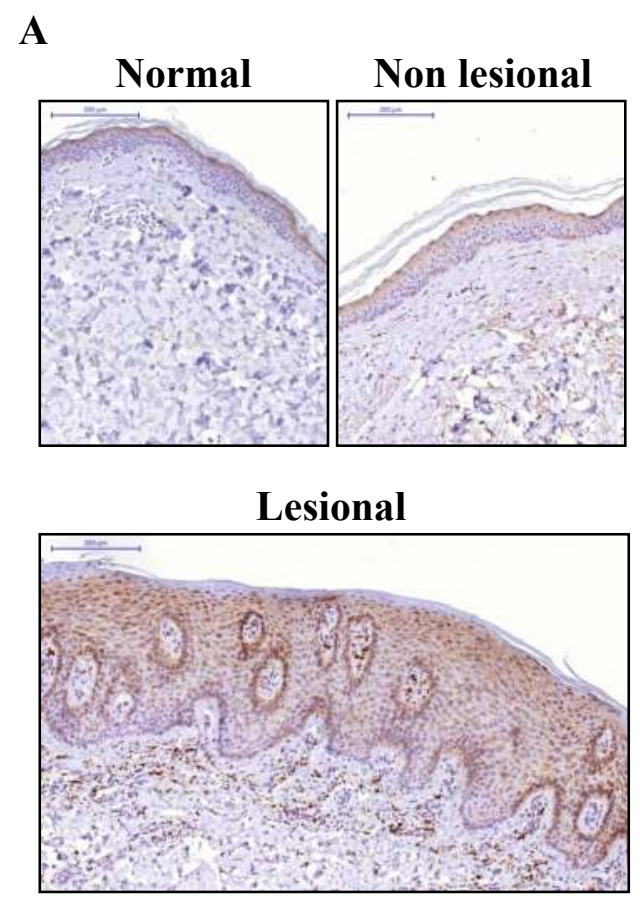

B

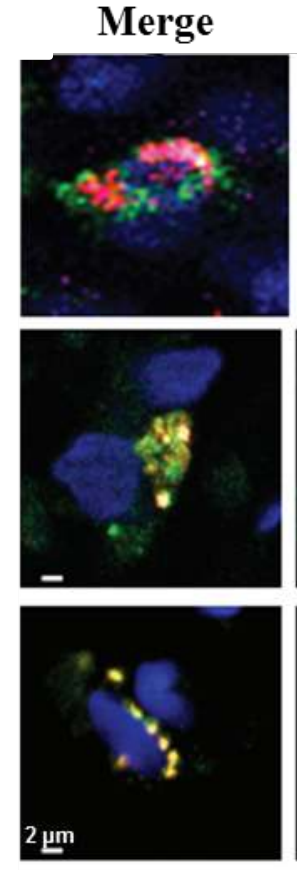

IL-17E

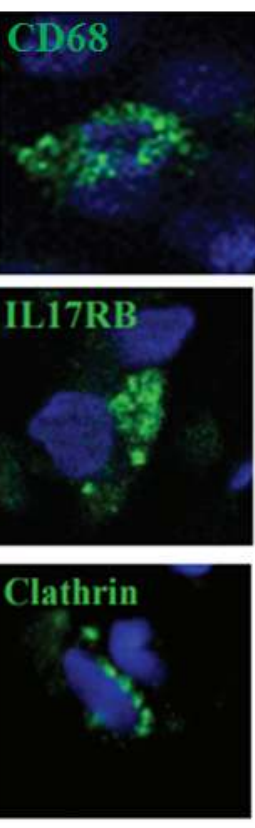

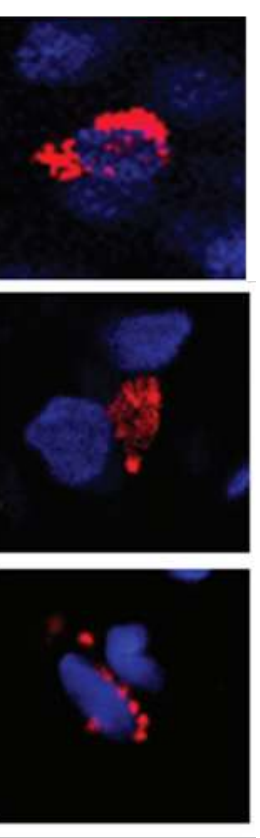
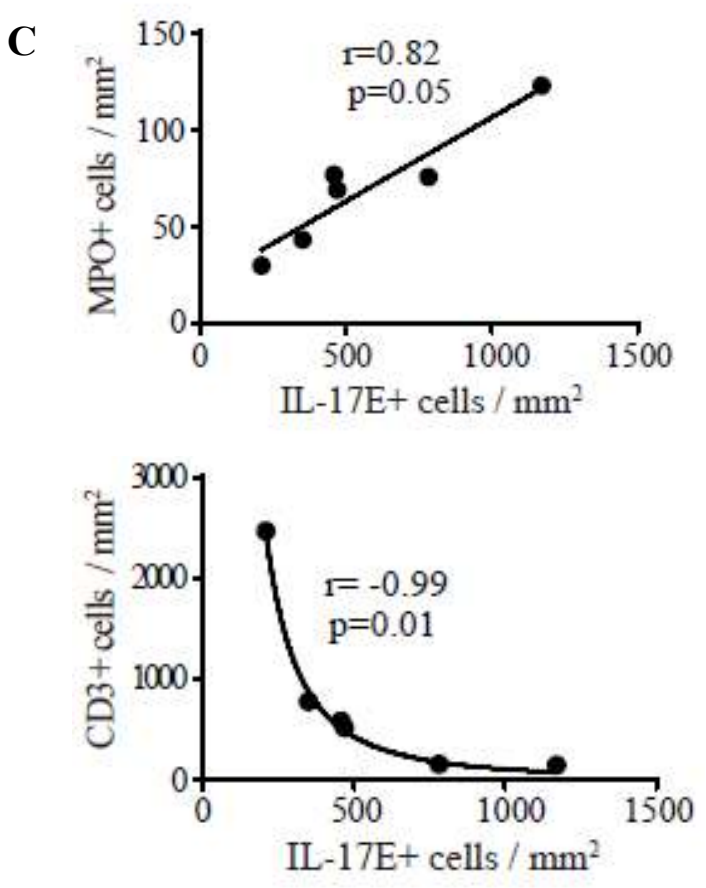

IL-17E activates M2 macrophages to produce IL-8 via p38 MAPK and recruit neutrophils

$\mathbf{A}$

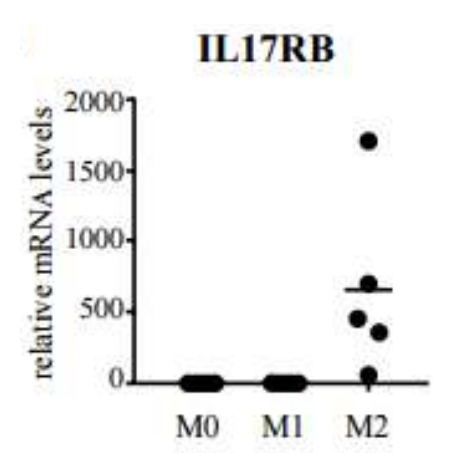

B

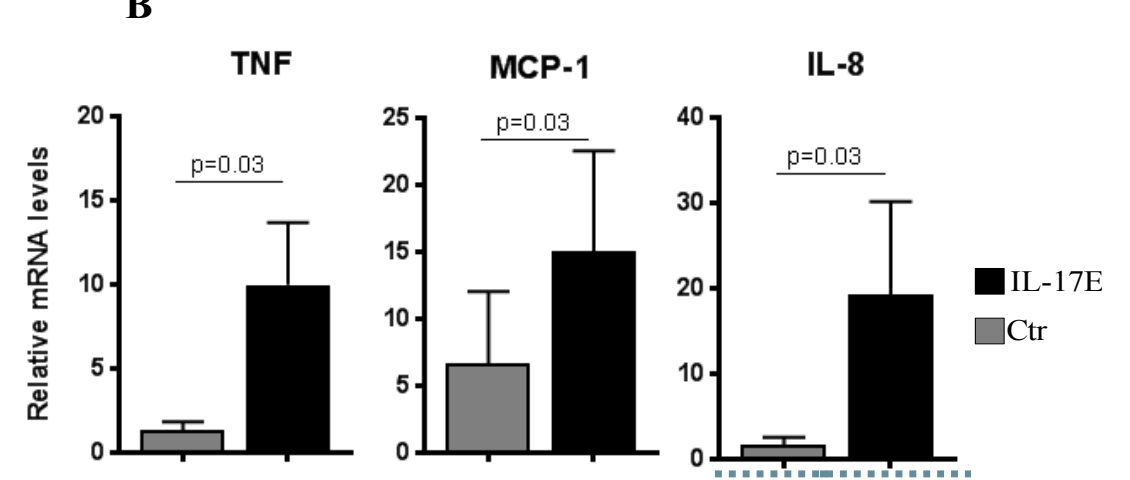

* No difference in the levels of IL-23, IL-12 and CCL22 (T-cell recruitment/polarization)

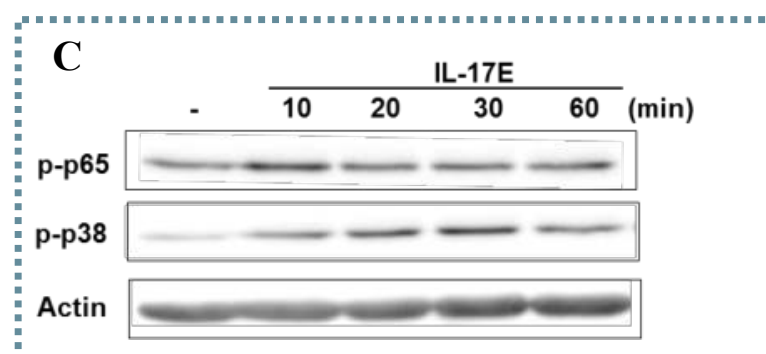

E
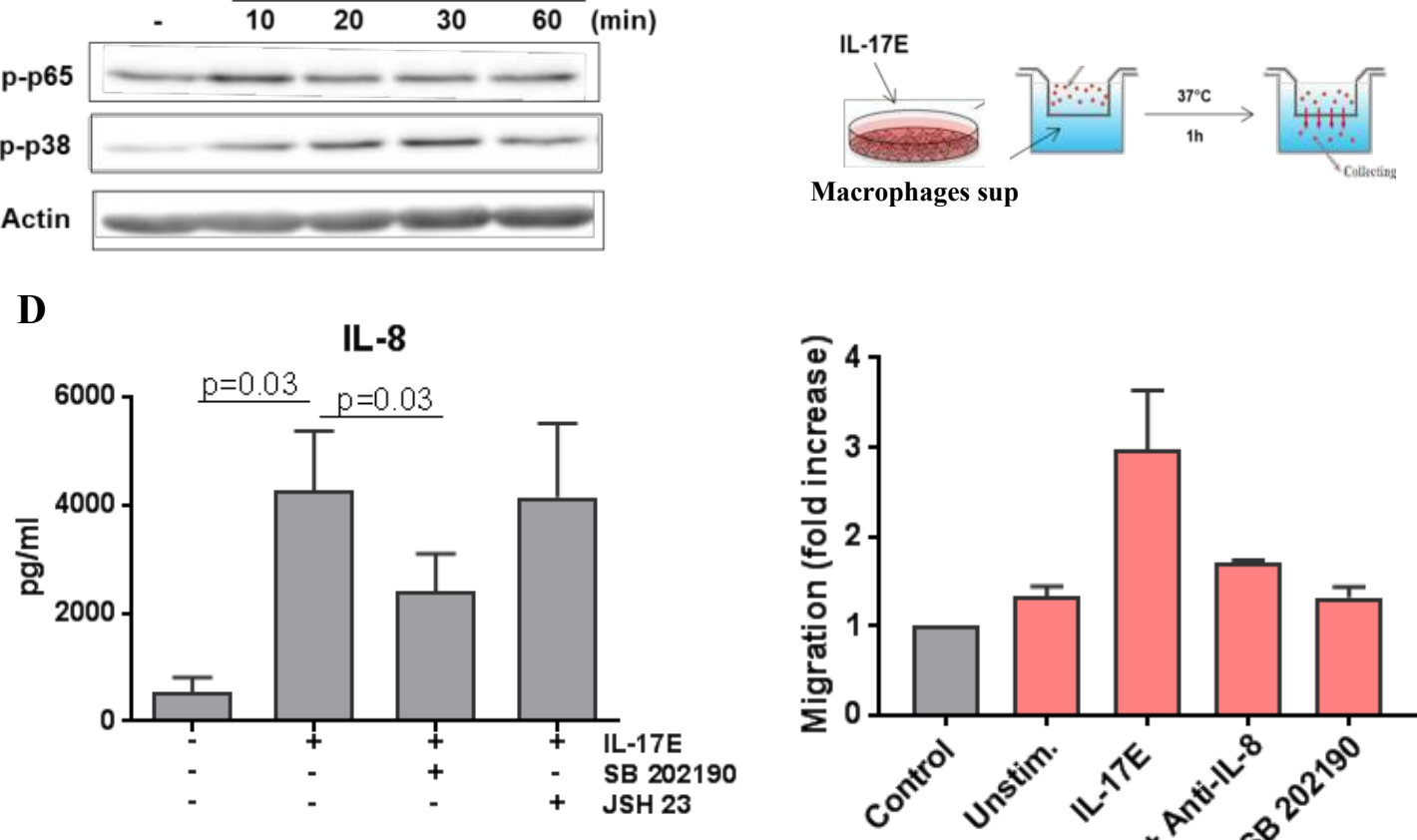

SB202190: p38 MAPK inhibitor JSH 23: NF-kB inhibitor

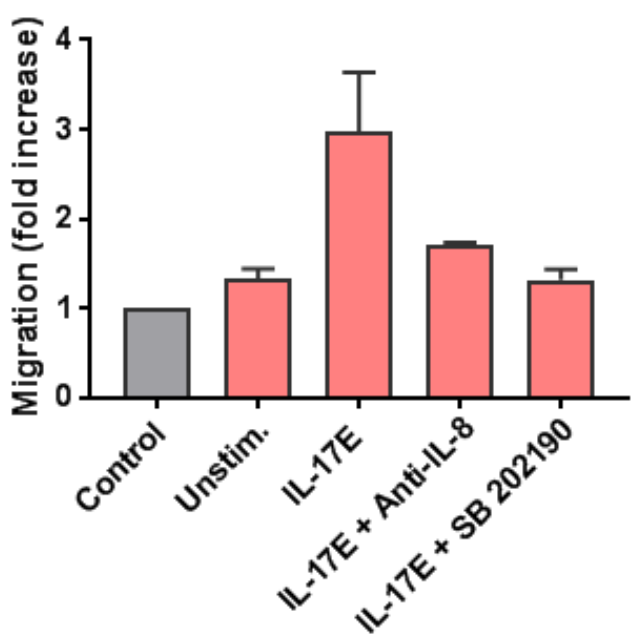

$\square$ Macrophage supernatants

IL-17E induces skin inflammation and the recruitment of neutrophils in healthy WT mice and in tape stripping model

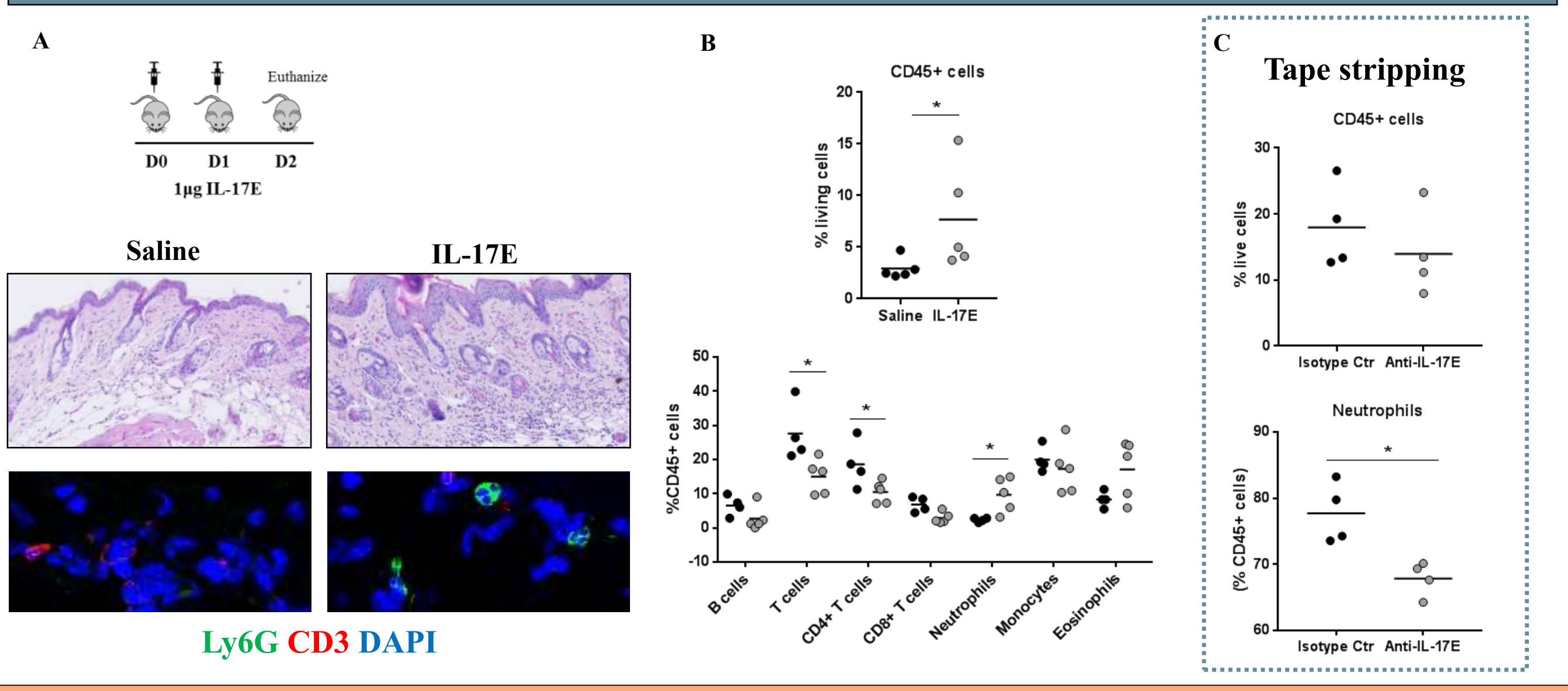

IL-17E induces the production of inflammatory cytokines and chemokines, in particular IL-8, in in-vitro M2 macrophages. IL-17E mediated-IL-8 production promotes neutrophil migration via p38 MAPK pathway. In vivo, IL$17 \mathrm{E}$ participates in skin inflammation and in the recruitment of innate immune cells, suggesting a pro-inflammatory role of this cytokine in psoriasis. 